\title{
Additive manufacturing of multi layered bioactive materials with improved mechanical properties: modelling aspects
}

\author{
KRZYZANOWSKI Michal ${ }^{1,2} \mathrm{a}^{*}$, SVYETLICHNYY Dmytro ${ }^{1, \mathrm{~b}}$ and BAJDA \\ Szymon ${ }^{1, c}$ \\ ${ }^{1}$ AGH University of Science and Technology, Mickewicza 30, 30-059 Krakow, Poland \\ ${ }^{2}$ Birmingham City University, Faculty of Computing, Engineering and the Built Environment, \\ Millennium Point, Curzon Street, Birmingham B4 7XG, UK \\ amichal.krzyzanowski@bcu.ac.uk, bsvetlich@metal.agh.edu.pl, csbajda@agh.edu.pl \\ ${ }^{*}$ corresponding author
}

\begin{abstract}
Keywords: Additive Manufacturing, Bioactive materials, Porosity, Crystallinity, Multilayered Laminate Structure, Selective Laser Melting, Finite element method, Lattice Boltzmann Method, Cellular Automata
\end{abstract}

\begin{abstract}
Multilayered laminate structures obtained by coating of ultrafine-grained metallic materials with bioactive and multifunctional composite coatings are considered for biomedical applications. Laser-assisted densification of multiple materials using laser cladding and selective laser melting is an alternative route to reduce the risk of early implant failure allowing for faster and cheaper fabrication. To understand the cooperative relationships between different factors that cam influence the manufacture of such bioactive laminates reflecting in their bioactivity and mechanical properties, the multi scale numerical modelling is applied. This work presents resent advances on development of integrated numerical models including generation, melting and solidification of the powder bed, considering surface flow, wettability, surface tension and other physical phenomena, specific mechanical and thermo-mechanical aspects and microstructure evolution.
\end{abstract}

\section{Introduction}

As bioinert material, Ti alloys are the most widely used to produce medical implants due to their exceptional mechanical properties, corrosion resistance and biocompatibility. There are two main problems with their application, namely: they contain alloying elements, such as $\mathrm{Al}$ and $\mathrm{V}$, which may be potentially toxic when released into human system [1] and their bonding to bone is not strong enough due to interfacial stability problems with the host tissues [2]. First issue may be resolved by replacing $\mathrm{Ti}$ alloys with commercially pure $\mathrm{Ti}$ (cpTi) having ultrafine-grained structure free of potentially toxic elements. The yield and also the ultimate strength of cpTi can exceed 1000MPa, which is even higher than the one of Ti alloys [1]. The bonding to bone of the titanium implant may be greatly enhanced by covering the titanium surface with some bioactive material able to form a biological bond with host tissue. One of the most important bioactive and bioresorbable materials are bioactive glasses and ceramics. Bioactive glasses, generally based on mixtures of $\mathrm{SiO}_{2}, \mathrm{CaO}, \mathrm{MgO}$, $\mathrm{Na}_{2} \mathrm{O}, \mathrm{K}_{2} \mathrm{O}$ and $\mathrm{P}_{2} \mathrm{O}_{5}$, due to their high bioactivity, osteoconduction and osteostimulation, are commonly used as a bone filling materials, small bone implants and for dental applications [3]. Ceramics and glasses are excellent bioactive or bioresorbable materials, but cannot be used alone due to intrinsic brittleness and relatively poor mechanical properties limiting their clinical applications to non-load bearing implants. Hence, they can be imposed as coatings on a mechanically tough nonbioactive metallic substrates to improve fixation and osteointegration of the whole implant. Additionally, a bioactive glass/ceramic coating protects both the substrate from corrosion and host tissues from corrosion products potentially causing some adverse reactions [4]. Designing a multilayered material system with mechanically tough metallic core is an alternative to reduce the risk of early implant failure. Refining the grain size through severe plastic deformation (SPD) is known to provide the processed metallic material with novel properties. The potential application of such 
advanced materials as biomedical implants has been evaluated by investigating the effect of different grain sizes on a variety of cell cell-substrate interactions. The refined to the nano- level grain surfaces are considered to have potential to alter absorption of proteins that mediate cell adhesion, control and enhance subsequent cell functions and tissue growth [5]. Combining any of SPD techniques used for grain refining at the surface layer of the material with subsequent thermomechanical (TMP) processing allows for obtaining metallic materials with multilayered bulk structure and an increased volume fraction of the nano-sized grains. Such duplex techniques have been successfully applied for bonding fcc material, such as 316L austenitic stainless steel, to achieve the enhanced yield and ultimate strength, while conserving an acceptable elongation to failure [6]. The multiscale numerical model based on finite element (FE) and coupled with cellular automata (CA) method have been developed recently to understand interface bonding mechanisms within such laminated structures, particularly the oxide scale behaviour and microstructure evolution [7]. Different aspects of the modelling of microstructure evolution around the oxidised interfaces are presented in this work.

Laser cladding using powder feeding is progressively considered in biomaterials research as the surface treatment approach conducted to calcium phosphate and bioactive glass coatings on $\mathrm{Ti}$ alloys $[8,9]$. Implants made by additive manufacturing methods can have increased longevity by providing more secure fixation using tailored porosity, which cannot be achieved by conventional manufacturing techniques. The potential is great. However, apart from the residual stresses due to mismatch in thermal expansion between metal, glass or ceramic, among the factors that determine bioactive effectiveness of the coated material are the morphological and microstructural features, such as surface roughness, porosity and crystallinity. The finite element (FE) based model of the laser cladding capable to predict several of the mentioned above phenomena is presented in this work.

The components currently manufactured by selective laser melting (SLM) can be made of different materials, both metallic and not metallic, such as ceramic and polymers. Following the rising demands for advanced biomedical applications, more sophisticated SLM techniques are also being intensively developed aiming for manufacturing multiple material components required for specific applications, where different materials with different properties are required at different locations [10, 11]. An appropriate application of physically based through process mathematical modelling capable to support the necessary quantitative analysis and optimization of the whole manufacturing process is currently in demand. The available multiscale models relies on combination of several models operating on different principles and (or) different scales. Most of the combinations are significantly heterogeneous multiscale numerical models that require complicated interfaces between different components eliminating the possibility of full scale modelling using a single numerical model without running the individual modules independently and combining them afterwards. Development of holistic models based entirely on one or two homogeneous methods will allow for both modelling very complex physical phenomena accompanying the manufacturing process and elimination of the complicated interfaces. The concept and the main assumptions of such holistic model based on CA and Lattice Boltzmann method (LBM) are presented elsewhere [12]. The modules considering particle movement, heat exchange and transfer, melting, wetting, oxidation effects, free flow of melted material and solidification developed to different extent and are also discussed in this work leaving some aspects for further consideration and analysis.

\section{Microstructure evolution around oxidised interfaces in bonding nanocrystallized metallic laminates using duplex techniques}

The main idea of the duplex techniques is combining of grain size refining processes based on SPD with a subsequent TMP with the aim of producing multilayered bulk structures with improved yield and ultimate tensile strengths in combination with an acceptable ductility. For instance, application of surface mechanical attrition treatment (SMAT) for refining the surface layer of the stainless steel plates up to a few hundred micron thickness following by bonding them together by using subsequent rolling or compression at elevated temperatures allows for significant improvement of mechanical properties of the bonded laminate structure (Fig. 1a). However, due to interfacial oxidation during the subsequent TMP the impurities deposited on the surface of the materials cause 
bonding imperfections. In addition, depending on the TMP parameters, either the oxide islets or continuous oxide layers can be developed at the interfaces. The oxide scale behavior during deformation becomes a part of microstructure evolution around the interfaces causing grain refinement and shear banding. Such shear banding can enhance bonding developing channels for the metal of the plates to come into contact. In addition to the temperature, texture and grain size, the difference in mechanical properties of oxide fragments and metal, and also dimensions of the oxide fragments, have been recognized as important technological factors. To understand, and importantly, to be able to predict the behaviour of the highly reactive interfaces during the processing of ultrafine grained or nanocrystallised multi-layered materials, the multiscale numerical model has recently been developed based on combination of FE and frontal cellular automata (CA) methods. The meso-level of the multiscale FE based model (Fig. 1b) is combined with the three-dimensional frontal CA numerical model in order to investigate appearance of new boundaries and rotation of sub-grains and grains around the oxidized interfaces during the subsequent straining [7]. Modelling the evolution of dislocation density and substructure arrangement is essential for the analysis of nanocrystalline materials. Based on principals of crystal plasticity, the evolution of dislocation density is calculated for every cell of the representative domain where the effects of deformation are influenced by dislocation motion on active slip systems and by distortion of the crystal lattice. The modelling approach is able to predict appearance of the low-angle boundaries due to growth of dislocation density when the density reaches its critical level (Fig $1 \mathrm{c}, \mathrm{d}$ ). The boundaries divide the grain into dislocation cells. The dislocation structure evolves at different rate due to differences in the slip rate for the different slip directions leading to rotation of the dislocation cells, sub-grains and grains during subsequent deformation.

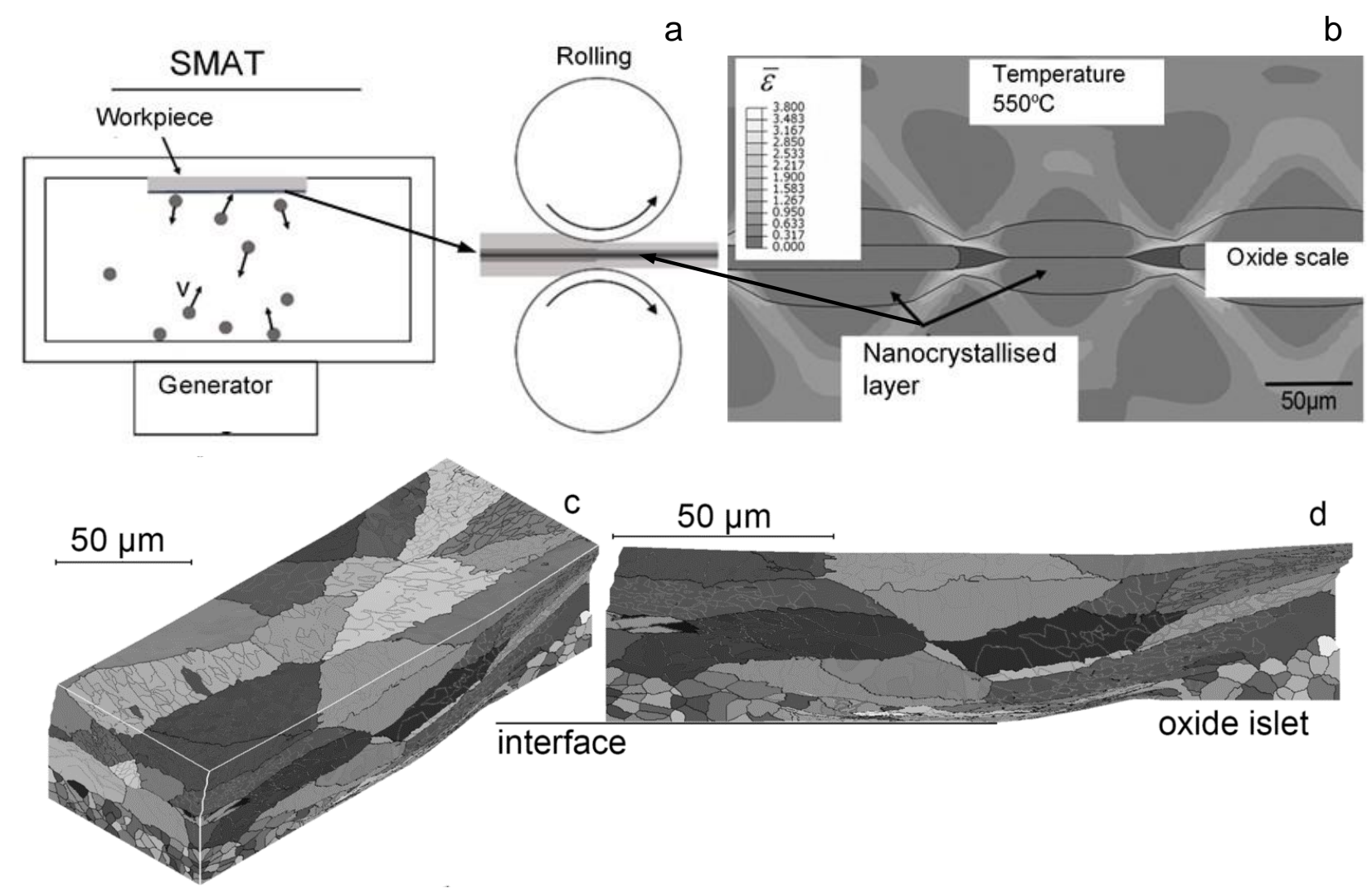

Fig. 1 Schematic representation of the duplex technique (a), the equivalent strain (b) and microstructure of $316 \mathrm{~L}$ steel $(\mathrm{c}, \mathrm{d})$ predicted around the oxidised interface after rolling 


\section{Evaluation of temperature and stress-strain distribution as well as crystallinity and porosity degree during laser cladding of bioactive material}

The sequentially coupled FE modelling approach has been used for the thermal stress calculations using ABAQUS/Standard software based on results of the thermal analysis. The transient temperature distributions, as an effect of the heat transfer from the moving laser beam, were used to calculate the transient strains and stresses induced in the heat affected zone (HAZ) [9]. The model consists of two sets of 3D elements. The first set represents the ultrafine-grained cpTi substrate while the second one represents 45S5 bioactive glass material accumulated on the base plate surface. In the experiential laser cladding process, the bioactive material is cladded gradually along with the laser beam movement. In the numerical modelling, increase of the amount of cladded bioactive material has been taken into account using a time-dependent activation of the finite elements, also known as the birth and death technique (Fig. 2a, b). At the beginning of the numerical analysis, the finite elements representing bioactive material have been deactivated. The element were selectively activated during the processing depending on the cladding speed. The laser beam is considered as a volumetric heat source defined by a Gaussian distribution to be absorbed in a volume of the representative domain.

Porosity, surface roughness and crystallinity are among the factors affecting the material bioactivity that have been modelled in this work (Fig. 2c). The high degree of crystallinity increases the onset time of generation of the hydroxyapatite surface layer, which is responsible for establishing a biological bond with host tissues. The crystallization process starts from nucleation and crystal growth and is modelled using well known Johnson-Mehl-Avrami-Kolmogorov equation. It is assumed in the model that both nucleation and crystal growth occur simultaneously. Porosity also affects the material bioactivity significantly improving its ability to bonding with host tissues. The overall porosity is calculated based on the initial density of the powder and the information about the sintering state of the material, the parameter known as the sintering potential. The sintering potential is defined by the sintering rate, surface energy, viscosity and the characteristic length scale of the cladded material. The details of the method applied in this work for calculation of the porosity, both overall and effective, as well as the level of crystallinity of the cladded bioactive material are described elsewhere [13].
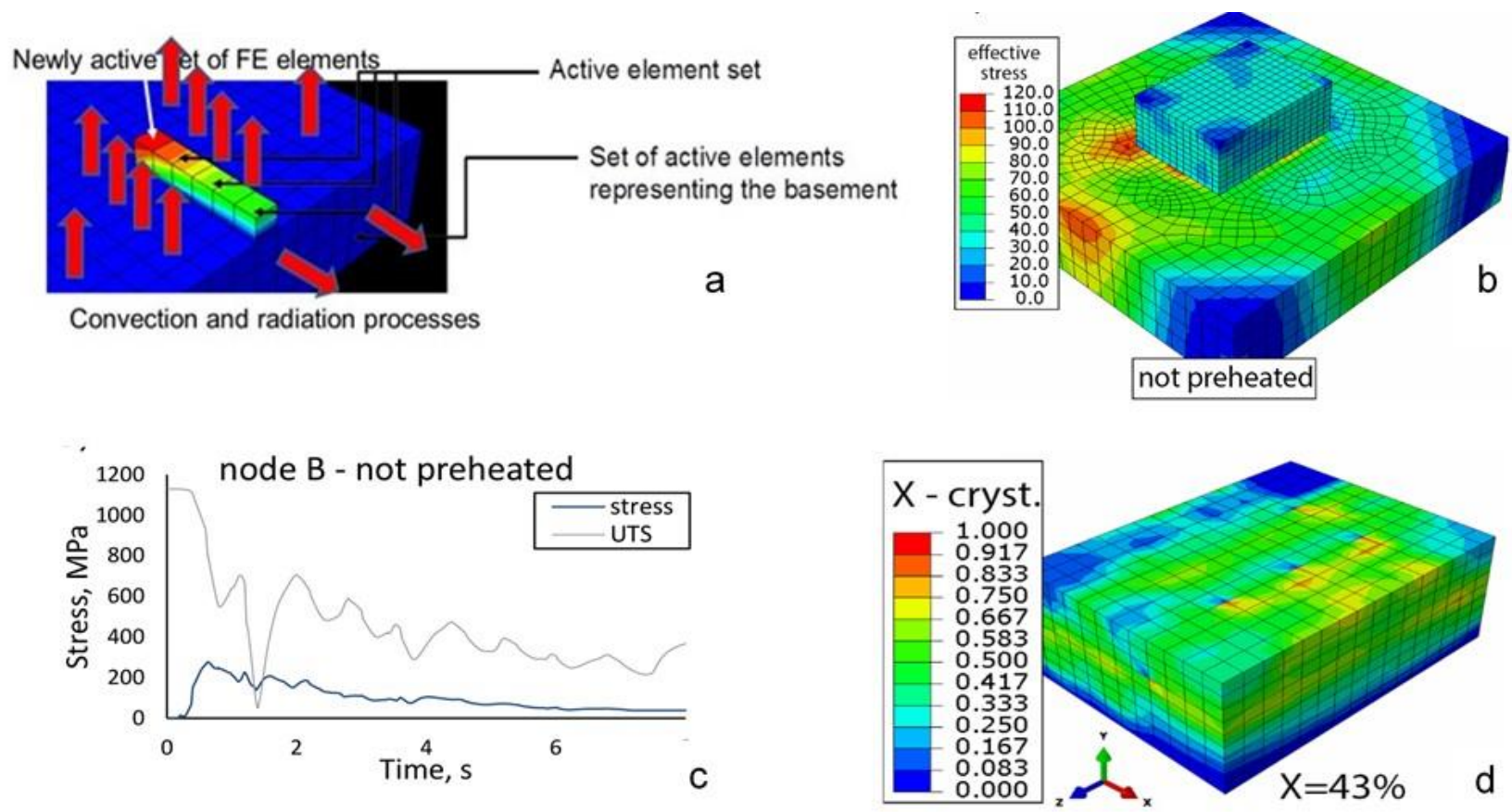

Fig. 2 Schematic representation of the time dependent activation and deactivation of the finite elements in the laser cladding modelling (a), predicted effective stress distribution after cladding on the not preheated basement (b), transient effective stress and UTS at node B on the substrate surface (c) and crystallinity distribution in bioactive glass predicted after cladding (d). 


\section{Modelling of energy transfer including solid-liquid phase transformation in SLM using CA- LMB numerical approach}

One can distinguish four main stages occurring simultaneously or sequentially in SLM powder bed based process. They include powder material delivery, heating and melting of the deposited powder, free or directed flow of the material in a liquid or semi-liquid state, and also cooling and solidification. The last three stages are accompanied by heat transfer between solid, liquid and gas phases and also by heat conduction. Throughout such technological process, powder deposition and laser treatment are repeated multiple times. These stages are represented by the physically based models considering main physical processes associated with SLM taking place in sequence or simultaneousely, such as particles movement in powder bed generation, laser beam heat exchange and transfer, melting, free flow of the melted material and solidification. The following numerical models have been developed recently, namely: powder bed generation model based on CA that is extended to particle movement during the process, heat exchange and transfer model based on LBM including laser treatment, phase transition model (solid-liquid) based on LBM and CA, and also model of fluid flow with free surface flow based on LBM. It is important that development of such holistic models based entirely on one or two homogeneous methods, such as CA and LBM, allows for modelling of very complex physical phenomena associated with the specific SLM process but eliminating interfaces between various components of the multiscale models at the same time. The presence of such complicated interfaces between models based on heterogeneous numerical methods almost eliminate the possibility of full scale modelling without running different models independently and combining them together afterwards rising serious limitations to such approaches. As it has been mentioned elsewhere [12], this holistic modelling platform is being developed inline with a homogeneous approach and represents combination of different sub-modules developed to different extent leaving many aspects of this integrated numerical approach for further consideration and analysis.

Nevertheless, the obtained results demonstrate certain possibilities and benefits of such approach for modelling of the entire SLM process characterised by melting and solidification of the deposited particulate material. In Fig. 3, the results of the simulation are presented for two consecutive time moments when the vertically orientated laser beam moves from the left to the right of the domain showing two layers of the deposited spherical particles. The particles are assumed almost opaque in the solid state and much more transparent when the state is changed from solid to liquid. The transparency is changed near the transformation temperature. The size of the melted pool depends on the power of the laser beam. The modelling space was $160 \times 100$ cells or nodes. Several sub-models have been recoded for parallel computations on GPU with CUDA technology using the graphic card GeForce 1060 with 1280 CUDA cores allowing for at least 100 times acceleration comparing with a sequential code.
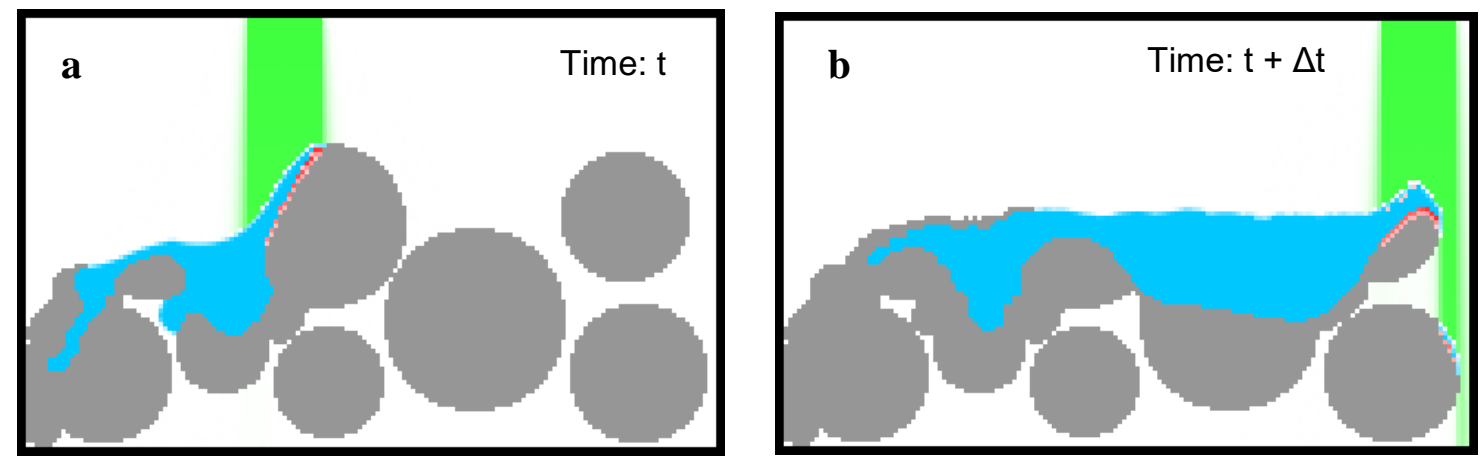

Fig. 3 Modelling results illustrating two consecutive time moments of SLM process showing two layers of the deposited spherical particles treated by the laser beam moving from the left to the right. 


\section{Acknowledgments}

This work was supported by the National Science Centre (NCN) in Poland, partly by the projects no. DEC-2013/09/B/ST8/00141) and no. UMO-2018/31/B/ST8/00622.

\section{References}

[1] C.N. Elias, M.A. Meyers, R.Z. Valiev, S.N. Monteiro, Ultrafine grained titanium for biomedical applications: An overview of performance, J. Mater. Res. Technol., 2(4) (2013) 340-350.

[2] Y. Ramaswamy, C. Wu, H. Zreiqat, Orthopedic coating materials: considerations and applications, Expert Rev. Med. Devices, 6(4) (2009) 423-430.

[3] V. Miguez-Pacheco, L.L. Hench, A.R. Boccaccini, Bioactive glasses beyond bone and teeth: Emerging applications in contact with soft tissues, Acta Biomater., 13 (2015) 1-15.

[4] F. Baino, E. Verné, Glass-based coatings on biomedical implants: a state-of-the-art review, Biomed. Glas., 3(1) (2017) 1-17.

[5] S. Bagherifard, R. Ghelichi, A. Khademhosseini, M. Guagliano, Cell response to nanocrystallized metallic substrates obtained through severe plastic deformation, ACS Appl. Mater. Interfaces 6 (2014) 7963-7985.

[6] L. Waltz, D. Retraint, A. Roos, P. Olier, Combination of surface nanocrystallization and corolling: creating multilayered nanocrystalline composites, Scr. Mater. 60 (2009) 21-24.

[7] S. Bajda, D. Svyetlichnyy, D. Retraint, M. Krzyzanowski, Numerical modelling of grain refinement around highly reactive interfaces in processing of nanocrystallised multi-layered metallic materials by duplex technique, Int. J. Adv, Manuf. Technol., 96(5-8) (2018) 2893-2905.

[8] R. Comesana, F. Quintero, F. Lusquinos et al., Laser cladding of bioactive glass coatings, Acta Biomater., 6 (2010) 953-961.

[9] M. Krzyzanowski, S. Bajda, Yijun Liu et al., 3D analysis of thermal and stress evolution during laser cladding of bioactive glass coatings, J. Mech. Behav. Biomed. Mater., 59 (2016) 404-417.

[10]L.E. Murr, Strategies for creative living: additively manufactured, open-cellular metal and alloy implants by promoting osseointegration, osteoconduction and vascularization: An overview, J. Mater. Sci. Technol., 35 (2019) 231-241.

[11]Ch. Wei, L. Li, X. Zhang, Y-H Chueh, 3D printing of multiple metallic materials via modified selective laser melting, CIRP - Annals - Manufacturing Technology, 67 (2018) 245-248.

[12]D. Svyetlichnyy, M. Krzyzanowski, R. Straka et al., Application of cellular automata and Lattice Boltzmann methods for modelling of additive layer manufacturing, Int. J. Num. Methods for Heat \& Fluid Flow, 28(1) (2018) 31-46.

[13]P. Kongsuwan, G. Brandal, Y. Lawrence Yao, Laser Induced Porosity and Crystallinity Modification of a Bioactive Glass Coating on Titanium Substrates, J. Manuf. Sci. Eng., 137(3), (2015) 031004. 